\title{
Evaluation of urgency in women, with a validated Urgency, Severity and Impact Questionnaire (USIQ)
}

\author{
Lior Lowenstein • Mary P. FitzGerald • \\ Kimberly Kenton • Lena Hatchett • \\ Ramon Durazo-Arvizu • Elizabeth R. Mueller • \\ Kara Goldman • Linda Brubaker
}

Received: 18 September 2008 / Accepted: 1 November 2008 / Published online: 20 November 2008

(C) The Author(s) 2008. This article is published with open access at Springerlink.com

\begin{abstract}
The objective of this study is to develop a validated, patient-oriented questionnaire to assess urgency and associated life impact. We interviewed six urogynecologists and urologists and five patients with overactive bladder $(\mathrm{OAB})$ symptoms about urinary urgency. Based on this data, we composed the first draft of our Urgency Severity and Impact Questionnaire (USIQ). A focus group of OAB patients provided feedback on the USIQ draft. The revised questionnaire has two parts: symptom severity (USIQ-S) and related quality of life (USIQ-QOL). The questionnaire was given to patients with clinically diagnosed $\mathrm{OAB}$ to establish face, content and discriminatory validity. Cronbach's alpha for the USIQ-S and USIQ-QOL were 0.85 and 0.90 , respectively. USIQ-QOL was moderately correlated with UDI-6 $(r=0.49, p<0.001)$, IIQ-7 $(r=$ $0.77, p<0.001)$, and OAB-q $(r=0.73, p<0.001)$. Mean
\end{abstract}

Financial support Pfizer Inc.

L. Lowenstein · M. P. FitzGerald · K. Kenton • E. R. Mueller ·

K. Goldman • L. Brubaker

Division of Female Pelvic Medicine and Reconstructive Surgery,

Department of Obstetrics and Gynecology and Urology,

Stritch School of Medicine, Loyola University Medical Center,

2160 South First Avenue, Bldg 104, Room 1004,

Maywood, IL 60153, USA

L. Hatchett $\cdot$ R. Durazo-Arvizu

Department of Preventive Medicine \& Epidemiology,

Stritch School of Medicine, Loyola University Medical Center,

2160 South First Avenue, Bldg 104, Room 1004,

Maywood, IL 60153, USA

L. Lowenstein $(\triangle)$

Female Pelvic Surgery and Reconstructive Surgery,

Rambam Medical Center, Faculty of Medicine,

Technion-Israel Institute of Technology,

Haifa, Israel

e-mail: lowensteinmd@gmail.com
USIQ-QOL and USIQ-S scores differed by clinical diagnosis. The USIQ is an easily understood questionnaire with adequate validity for use in clinical practice and research.

Keywords Urgency - OAB - Lower urinary tract . Questionnaire $\cdot$ Health related quality of life

\section{Introduction}

The International Continence Society (ICS) defines urinary urgency as "the complaint of a sudden compelling desire to pass urine that is difficult to defer." [1]. As currently defined, urgency describes an abnormal sensation that is distinguishable from the normal feeling of "urge" to void, which occurs during a normal bladder-filling cycle. Attempts to measure urgency are confounded by difficulties in understanding its definition and the context of the normal urge to void. Although urinary urgency is a key symptom of overactive bladder $(\mathrm{OAB})$, most $\mathrm{OAB}$ research concentrates on urinary incontinence and urinary frequency. This may be partly due to the controversy surrounding the term 'urgency'. The aim of our current study was to develop and validate a urinary urgency questionnaire to measure the severity and quality of life (QOL) impact from urinary urgency, in order to advance the clinical understanding of urinary urgency, and ultimately to guide the evaluation and treatment of patients with $\mathrm{OAB}$.

\section{Materials and methods}

Ethical approval for all aspects of this research was granted by our Institutional Review Board. Developing the USIQ was a multi-step process (Fig. 1). 
Fig. 1 Overview of the validation of the USIQ

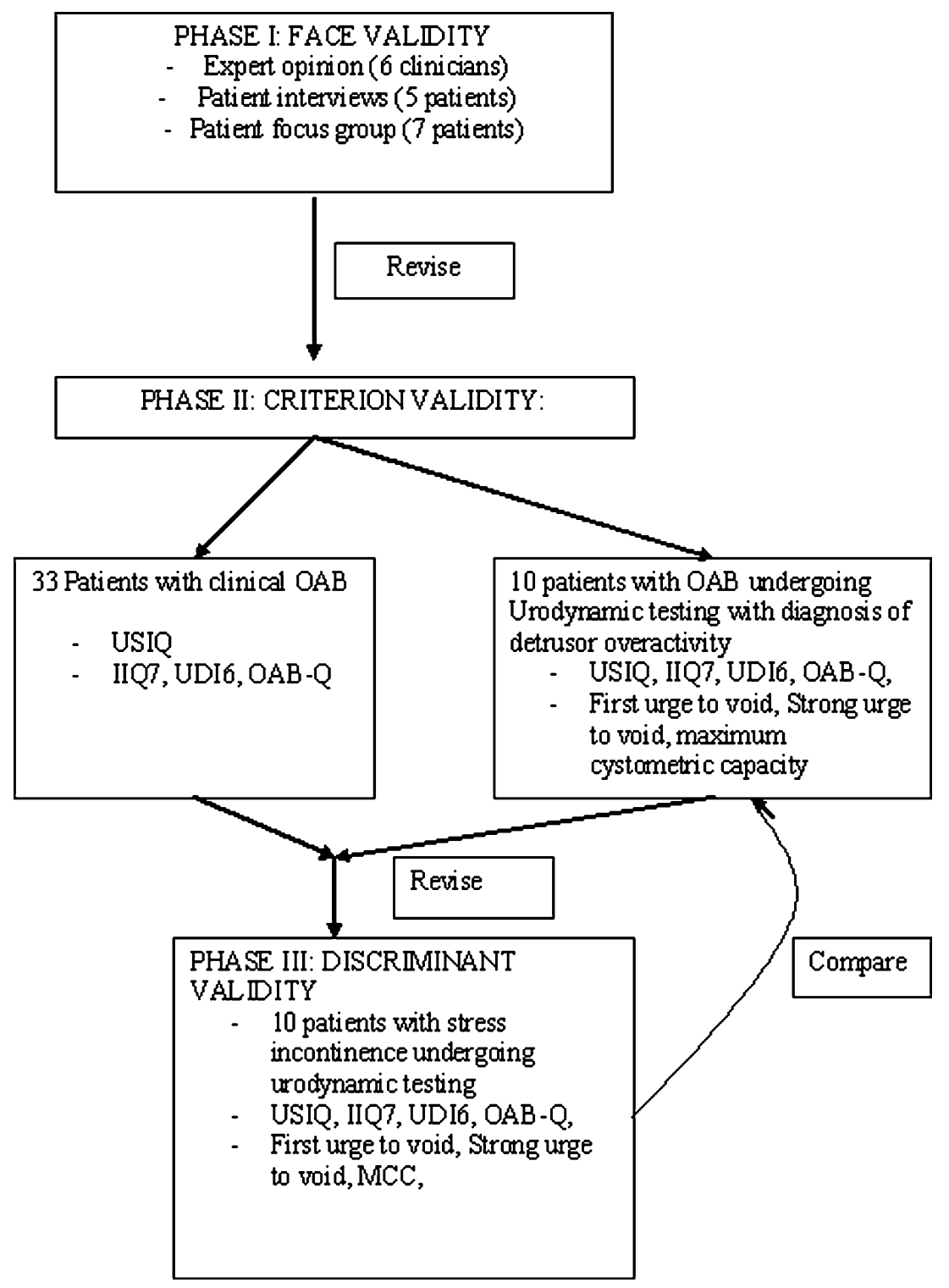

Instrument development

We composed the first draft of the USIQ questionnaire after interviewing six urogynecologists and urologists across the United States with expertise in the evaluation and treatment of OAB. Experts offered their opinions and perceptions regarding important aspects (domains) of patients' experiences of urinary urgency and associated bother.

Next, we recruited five patients with OAB and symptoms of urge incontinence based on clinical diagnosis, from our referral urogynecology clinic. Consenting patients met one-on-one with a psychologist (LH), who explored and characterized their experience of urinary urgency, including descriptions of its severity and effects on activities of daily living. Patients again offered their opinions and perceptions about their experiences of urinary urgency and associated bother.
Important domains thus identified were used to construct the initial version of the USIQ, which consisted of two parts with an initial filter question, designated for self-administration. We utilized the ICS definition for urgency for this filter question. To ensure patients related their responses only to urgency symptoms, we added the following paragraph to our written instructions "The following questions are only about your experience of urinary urgency. Not about other urinary symptoms"

The first part, the Urgency Severity Questionnaire(USIQ-S), consisted of eight items which inquired about urgency symptoms and severity; the second part, the Urgency Impact Questionnaire-Quality of Life (USIQQOL), consisted of seven questions adopted and modified from the widely recognized and accepted Incontinence Impact Questionnaire (IIQ-7) [2]. The IIQ was found to capture all the domains which brought up by both the 
patients and the experts. Each item of the bother from urgency was measured originally using two different scales: (1) a categorical scale similar to the existing IIQ-7 and (2) a ten-point Likert scale with ' 0 ' indicating no bother at all, and ' 10 ' indicating maximum bother.

Face validity

A psychologist (LH) then led a focus group of seven women recruited from our urogynecology clinic. All participants had a clinical diagnosis of $\mathrm{OAB}$ and reported urge incontinence on the Medical Epidemiological and Social Aspects of Aging questionnaire (MESA) [3]. Focus group participants reviewed the USIQ draft and each participant individually completed and added written comments on the specific USIQ items. Next, the group together discussed issues related to symptoms of urgency, the impact of urgency on patients' lives and feedback on the ability of the USIQ to capture urgency symptoms. Based on the response of our focus groups, a question about the impact of urgency on sexual function was added to the USIQ-QOL.

\section{Criterion validity}

Criterion validity was established by measuring the strength of the relationship between the USIQ-S and USIQ-QOL score(s) and existing, validated measures of OAB symptom severity. Since there is no gold standard measure of the severity of urinary urgency, we explored the magnitude of the associations between the USIQ scores and the validated Urogenital Distress Inventory (UDI-6) [2], Incontinence Impact Questionnaire (IIQ-7) [2], and Overactive Bladder symptom and healthrelated quality of life questionnaire (OAB-Q) [4]. Forty-three patients with a clinical diagnosis of $\mathrm{OAB}$, ten of whom also had urodynamically confirmed DOI, were recruited from our outpatient clinic to take part in this phase of the study. Consenting participants provided demographic data and completed the IIQ7, UDI6, OAB-Q, and USIQ. Criterion validity was established by correlating urodynamic parameters and responses to the different questionnaires.

\section{Discriminant validity}

Discriminant validity was established by asking patients without a clinical diagnosis of $\mathrm{OAB}$ to complete the same measures at the time of urodynamic testing. Ten patients with urodynamic stress incontinence (USI) who did not have urge incontinence (they did not respond positively to any urge item on MESA-urge subscale) provided demographic data and completed the USIQ, IIQ7, UDI6, OABQ. Participants were asked to complete the USIQ questionnaire even if they did not have urgency symptoms and the answer for the first question was 'No'. We aimed to establish that USIQ score(s) are higher among patients with OAB than patients with USI.

\section{Statistical analysis}

Decision rules for item reduction were (1) low-item to total correlations $(<0.4)$; or $(2)$ inadequate factor loading on any factor $(<0.4)$. All items on each USIQ subscale were summed to score each USIQ scale. The mean value for USIQ-S and USIQ-QOL items (item range $0-4$ ) was calculated, and multiplied by 25 to achieve a range of 0 to 100 . Items which were responded as 'unknown' were recorded as missing values. Higher scores indicate more severe urgency symptoms and greater impact on quality of life respectively. Cronbach's alpha was used to assess the internal consistency of the USIQ-S and USIQ-QOL. An average Cronbach's alpha of $0.7-0.8$ is generally considered acceptable for demonstration of internal consistency [5]. However, a very high alpha above 0.9 suggests highlyrelated items, and item redundancy. Pearson's correlation coefficient was used to compare responses to the USIQ-S and USIQ-QOL instruments to IIQ-7, UDI-6, OAB-Q, scores and urodynamic parameters; first desire to void, strong desire to void, and maximum cystometric capacity (MCC). Discriminatory validity was ascertained by comparing USIQ scores between clinical groups (controls without urgency versus patients with $\mathrm{OAB}$ ) using a twosample $t$-test and the area under the Receiver Operating Characteristic (ROC) curve. Exploratory factor analysis was applied to estimate factor loading and to determine the individual contribution of each item to the composite score. A 0.05 significance level was used for all statistical tests. No one-sided tests were done.

\section{Results}

Fifty three women with a mean age of 56 (29-87) years participated in this study. Most participants were Caucasian $(87 \%)$.

Item reduction

We omitted the ten-point Likert scale questions leaving five symptom severity items and eight QOL items due to the high correlation between responses, on the five-point categorical HRQOL scale and the ten-point Likert scale to bother from urgency items, $(r=0.89, p<0.001)$; the lower discriminatory power of the ten-point Likert scale questions, measured by the area under the ROC curve $(0.82$ versus 0.77 , respectively); and the simplicity of the categorical scale. All items had good loading scores ranging from 0.48 to 0.85 (Table 1). 
Table 1 Exploratory factor analysis and correlation between specific items with total USIQ subscale score

\begin{tabular}{|c|c|c|c|}
\hline Item analysis & Factor loading & $\begin{array}{l}\text { Correlation with } \\
\text { total score (rho) }\end{array}$ & $\mathrm{P}$ value \\
\hline \multicolumn{4}{|l|}{ USIQ-S } \\
\hline During the last month what percentage of urinations had urgency? & 0.71 & 0.79 & 0.0001 \\
\hline When you have urgency is it typically... & 0.76 & 0.83 & 0.0001 \\
\hline How long can you wait once you have urgency? & 0.47 & 0.65 & 0.0001 \\
\hline During the last month how much did urinary urgency bother you? & 0.86 & 0.87 & 0.0001 \\
\hline In an average 24 hours how often was urinary urgency present? & 0.86 & 0.87 & 0.0001 \\
\hline \multicolumn{4}{|l|}{ 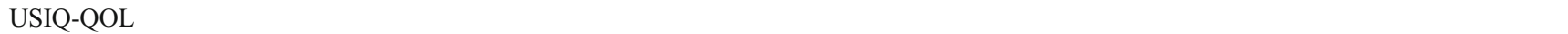 } \\
\hline Ability to do household chores, work? & 0.72 & 0.71 & 0.0001 \\
\hline Ability to do physical activity such as walking, swimming or other exercise? & 0.67 & 0.82 & 0.0001 \\
\hline Ability to have an intimate relationship or sexual intercourse? & 0.52 & 0.62 & 0.0001 \\
\hline Entertainment activities such and going to movie or concert? & 0.92 & 0.85 & 0.0001 \\
\hline Ability to travel by car or bus for a distance greater than 30 minutes away from home? & 0.82 & 0.85 & 0.0001 \\
\hline Participating in social activities outside your home? & 0.80 & 0.85 & 0.0001 \\
\hline Emotional health (nervousness, depression, etc)? & 0.57 & 0.64 & 0.0001 \\
\hline Feeling frustrated? & 0.8 & 0.81 & 0.0001 \\
\hline
\end{tabular}

Internal consistency for the USIQ-S and USIQ-QOL as measured by Cronbach's alpha was excellent, 0.85 and 0.90 , respectively. USIQ-S and USIQ-QOL scores moderately correlated $(r=0.69, p<0.001)$, thus supporting the association between the two parts of the questionnaire.

After revision of the USIQ based on focus group feedback and analysis described above, an optimized version of the questionnaire with a reduced number of items and scoring methods for both urgency severity and for its impact on quality of life was obtained (Appendix A).

\section{Criterion validity}

USIQ-QOL, which measures the effect of urgency on HRQOL, correlated moderately with existing, validated HRQOL questionnaires; UDI-6 $(r=0.49, p<0.001)$, IIQ-7 $(r=0.77, p<0.001)$ and OAB-q $(r=0.73, p<0.001)$. Similar correlations were found between the USIQ-S and UDI-6 $(r=0.34, p<0.02)$, IIQ-7 $(r=0.42, p<0.002)$, and OAB-q $(r=0.73, p<0.001)$. Except for a strong inverse correlation between USSBQ-QOL and the volume at which patients had strong desire to void $(r=-0.81, p<$ 0.015), neither USIQ-S nor USIQ-QOL scores correlated with other urodynamic parameters.

\section{Discriminant validity}

Mean USIQ-QOL and USIQ-S scores differed by clinical diagnosis. OAB patients had higher mean USIQ-S and USIQ-QOL scores than patients with USI ( $66 \pm 18$ vs. $34 \pm$ $24, p<0.001$ and $48 \pm 22$ vs. $19 \pm 25, p<0.005$, respectively). The area under the ROC curve, which was used to assess "urgency" discrimination, was 0.87 and 0.82 for USIQ-S and USIQ-QOL, respectively.

\section{Discussion}

We present a new questionnaire which has excellent internal consistency, good construct, face and discriminatory validity, and effectively captures the severity of urgency and its impact on quality of life. The USIQ addresses items which are important to patients with symptoms of urgency regardless of their continence status.

As expected, we found a moderate correlation between severity of urgency symptoms and their effect on patients' quality of life. This is particularly important when evaluating whether an individual will benefit from a treatment for symptoms of urgency and for evaluating the efficacy of such treatment.

Previous published questionnaires evaluate urgency symptoms as part of the evaluation of $\mathrm{OAB}$, including day time frequency, nocturia, urgency, and urgency incontinence. The Overactive Bladder Symptom Score (OABSS) [6], has been validated in a cohort of Japanese patients. It measures overall symptom severity due to day time frequency, nocturia, urgency, and urge incontinence. Urgency is evaluated in this questionnaire, by the following question "How often do you have a sudden desire to urinate, which is difficult to defer?". The OABSS total score $(0-15)$ is obtained by simple summation of the four individual symptoms [6]. Validity and reliability was demonstrated to be good as it was tested for the total score. The severity of urgency is evaluated only by one question which inquires about the frequency that patient has urgency symptoms. Warning time and the severity of the urgency symptoms are not captured by this questionnaire.

The Overactive Bladder Questionnaire (OAB-Q) [4] and the primary $\mathrm{OAB}$ symptom Questionnaire (POSQ) [7] are validated questionnaires which evaluate $\mathrm{OAB}$ symptoms and 
related bother. The OAB-Q [4] has a second part which assesses HRQOL in both continent and incontinent women. Both questionnaires have specific items which inquire about urge symptoms. A possible limitation of these questionnaires is using the term "urge" rather "urgency" as this may confuse patients who use the term urge for describing strong desire to void. In our new questionnaire, we used the ICS definition of urgency, and the results of our study suggest that the questionnaire was comprehended by all participants.

The Indevus Urgency Severity Scale (IUSS) is a validated questionnaire which evaluates the severity of urgency using a single question [8]. The questionnaire was validated on large population-based sample. Its main limitation is the use of a non-ICS definition of urgency [1], "Degree of urgency is meant to describe your urge to urinate", a mixing of the terms "urge" and "urgency" that may lead to confusion. Using only one question may also limit the ability of the questionnaire to capture other dimensions of urgency.

The Urgency Perception Score (UPS) was also found to be a validated and reliable tool for evaluating urgency symptoms [9]. The strength of this new tool is its ability to score urgency sensation on a continuous scale with different grades of severity. As was recognized by the authors, a limitation of this questionnaire is that validation in males and females was not done separately. The authors acknowledged that the two sexes may have different etiologies for urgency and urgency may be perceived in a different way. In addition, the UPS questionnaire does not evaluate the effect of urgency symptoms on patients' quality of life.

Finally, a new validated urgency questionnaire was published by Al-Buheissi et al. [10]. This questionnaire contains ten items which evaluates urgency severity and urge incontinence symptoms. The main limitation of this questionnaire is that its scoring system does not differentiate between symptoms of urgency and urge incontinence. Moreover, the terms urgency and urge are used interchangeably [10]. The USIQ evaluates urgency symptoms independent of urge incontinence. In order to ensure that patients related their responses only to urgency symptoms, we emphasized in our written instruction of the questionnaire that the questions inquire about urgency symptoms and not about other lower urinary tract symptom.

In conclusion, the USIQ is an easily understood questionnaire with adequate validity for use in research and in clinical practice to describe the severity and life impact of urinary urgency. Future testing will be needed to evaluate the stability (test-retest reliability) and sensitivity to change following treatment for urgency symptoms.

\section{Conflicts of interest None.}

Open Access This article is distributed under the terms of the Creative Commons Attribution Noncommercial License which permits any noncommercial use, distribution, and reproduction in any medium, provided the original author(s) and source are credited. 


\section{Appendix A}

The following questions are only about your experience of urinary urgency, NOT about other urinary symptoms.

1. During the last month, what proportion of your urinations had urgency associated with them?

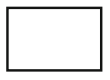

None or almost none of the urinations

Some of the urinations
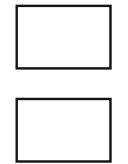

About half of the urinations

Most of the urinations

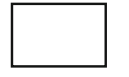

All or almost all of the urinations

Don't know

2. When you have urgency, is it typically

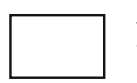

Extremely Mild

Mild

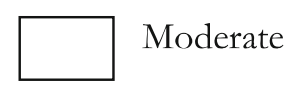

Severe $\square$ Extremely Severe

Don't know

3. Check the best answer for how long can you wait to urinate once you have urgency.

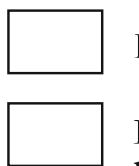

Half an hour or more

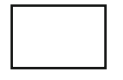

Less than half an hour but more than 5 minutes

Less than 5 minutes but more than 1 minute

I cannot wait at all

Less than 30 seconds
Don't know

4. During the last month, how much has urinary urgency bothered you?
$\square$ None
$\square$ Moderate
$\square$
Extensive
Slight

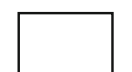
Considerable
Don't know

5. In an average 24 hour period how much of the time is urinary urgency present?
$\square$
Never

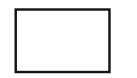
Sometimes
Always
$\square$ Rarely
Usually
Don't know 
II. The following statements are about the impact of urinary urgency on your life.

\begin{tabular}{|c|c|c|c|c|c|}
\hline $\begin{array}{l}\text { This question } \\
\text { does not apply } \\
\text { to me }\end{array}$ & Not At All & Somewhat & Moderately & $\begin{array}{l}\text { Quite } \\
\text { A Bit }\end{array}$ & Always \\
\hline
\end{tabular}

1 How much does urinary urgency affect your: Ability

to do household chores, do your work or schoolwork?

2 How much does urinary urgency affect your:

Ability to do physical activities such as walking,

swimming or other exercise?

3 How much does urinary urgency affect your: Ability

to have an intimate relationship or sexual intercourse?

4 How much does urinary urgency affect your:

Entertainmnetactivities such as going to a movie or concert?

5 How much does urinary urgency affect your:

Ability to travel by car or bus for a distance greater

than 30 minutes away from home?

6 How much does urinary urgency affect your:

Participating in social activities outside your home?

7 How much does urinary urgency affect your:

Emotional health (nervousness, depression, etc)?

8 How much does urinary urgency affect your:

Feeling frustrated?

\section{References}

1. Abrams P, Cardozo L, Fall M, Griffiths D, Rosier P, Ulmsten U et al (2002) The standardisation of terminology of lower urinary tract function: report from the Standardisation Sub-committee of the International Continence Society. Neurourol Urodyn 21:167178

2. Uebersax JS, Wyman JF, Shumaker SA, McClish DK, Fantl JA (1995) Short forms to assess life quality and symptom distress for urinary incontinence in women: the Incontinence Impact Questionnaire and the Urogenital Distress Inventory. Continence Program for Women Research Group. Neurourol Urodyn 14:131-139

3. Herzog AR, Diokno AC, Brown MB, Normolle DP, Brock BM (1990) Two-year incidence, remission, and change patterns of urinary incontinence in noninstitutionalized older adults. J Gerontol 45:M67-74

4. Coyne K, Revicki D, Hunt T, Corey R, Stewart W, Bentkover J (2002) Psychometric validation of an overactive bladder symptom and health-related quality of life questionnaire: the OAB-q. Qual Life Res 11:563-574
5. Altman D (1991) Practical Statistics for Medical Reseach. In London, Chapman and Hall

6. Homma Y, Yoshida M, Seki N, Yokoyama O, Kakizaki H, Gotoh M (2006) Symptom assessment tool for overactive bladder syndromeoveractive bladder symptom score. Urology 68:318-323

7. Matza LS, Thompson CL, Krasnow J, Brewster-Jordan J, Zyczynski T, Coyne KS (2005) Test-retest reliability of four questionnaires for patients with overactive bladder: the overactive bladder questionnaire (OAB-q), patient perception of bladder condition (PPBC), urgency questionnaire (UQ), and the primary $\mathrm{OAB}$ symptom questionnaire (POSQ). Neurourol Urodyn 24:215-225

8. Nixon A, Colman S, Sabounjian L, Sandage B, Schwiderski UE et al (2005) A validated patient reported measure of urinary urgency severity in overactive bladder for use in clinical trials. J Urol 174:604-607

9. Blaivas JG, Panagopoulos G, Weiss JP, Somaroo C, Chaikin DC (2007) The urgency perception score: validation and test-retest. J Urol 177:199-202

10. Al-Buheissi S, Khasriya R, Maraj BH, Malone-Lee J (2008) A simple validated scale to measure urgency. J Urol 179:1000-1005, discussion 1005 